\title{
O ÍNDICE ESPLÊNICO COMO PREDITOR DE SANGRAMENTO E RECIDIVA VARICOSA NO SEGUIMENTO TARDIO DE PACIENTE ESQUISTOSSOMÓTICOS APÓS TRATAMENTO ENDOSCÓPICO EXCLUSIVO
}

\author{
THE SPLENIC INDEX AS PREDICTOR OF BLEEDING AND VARICEAL RECURRENCE IN THE LATE FOLLOW-UP OF \\ SCHISTOSOMOTIC PATIENTS AFTER EXCLUSIVE ENDOSCOPIC TREATMENT
}

Alexandre BORGHERESI ${ }^{\oplus}{ }^{\oplus}$, Ramiro COLLEONI ${ }^{\oplus}{ }^{\oplus}$, Milton SCALABRINI ${ }^{\oplus}$, David SHIGUEOKA ${ }^{2} \odot$

RESUMO - RACIONAL: O tratamento endoscópico das varizes esofágicas tem sido utilizado como principal intervenção em pacientes com hipertensão portal secundária à esquistossomose, mas com taxas significativas de recorrência de varizes esofágicas e ressangramento. Os resultados em longo prazo do tratamento endoscópico exclusivo são pouco estudados quanto à relação das dimensões esplênicas neste contexto. OBJETIVO: Avaliar, por meio da ultrassonografia, o índice esplênico e a dimensão longitudinal (craniocaudal) do baço como preditores de ressangramento e recorrência de varizes no seguimento tardio de pacientes esquistossomóticos não operados, após erradicação endoscópica das varizes esofágicas. MÉTODOS: Estudo observacional retrospectivo por meio da análise de prontuários de pacientes com diagnóstico de esquistossomose hepatoesplênica. A curva ROC foi usada para determinar o melhor ponto de corte para o índice esplênico médio como preditor de recorrência e sangramento. RESULTADOS: Foram analisados 54 pacientes, durante o período de 2002 a 2018. O tempo médio de seguimento foi de 8 anos. O índice esplênico provou ser um teste sensível em valores acima de 144 como preditor de ressangramento. Na análise da dimensão longitudinal, o valor acima de $20 \mathrm{~cm}$ apresentou teste estatisticamente significativo para recorrência de varizes e valor acima de $19 \mathrm{~cm}$ apresentou-se como teste muito sensível e estatisticamente significativo para ressangramento. CONCLUSÃO: A análise do índice esplênico e da dimensão craniocaudal, obtidos por ultrassonografia, podem predizer recorrência de varizes e ressangramento após erradicação endoscópica exclusiva.

DESCRITORES: Esquistossomose. Hipertensão Portal. Varizes Esofágicas e Gástricas. Hiperesplenismo. Endoscopia Gastrointestinal

ABSTRACT - BACKGROUND: Endoscopic treatment for esophageal variceal has been used as the main intervention in patients with portal hypertension secondary to schistosomiasis, but with significant rates of recurrence of esophageal variceal and rebleeding. The long-term results of exclusive endoscopic treatment are poorly studied as the relationship of the splenic dimensions in this context. AIM: The aim of this study was to identify, through ultrasonography, whether the splenic index and the longitudinal (craniocaudal) dimension of the spleen are the predictors of rebleeding and variceal recurrence in late follow-up of patients with nonoperated schistosomiasis, after endoscopic eradication of esophageal variceal. METHODS: This is a retrospective and observational study analyzing the medical records of patients diagnosed with hepatosplenic schistosomiasis. The receiver operating characteristic curve was used to determine the best cutoff point for the mean splenic index as a predictor of recurrence and bleeding. RESULTS: A follow-up of 54 patients were analyzed during the period from 2002 to 2018 . The mean follow-up time was 8 years. The splenic index with value $>144$ was proved to be a sensitive test for rebleeding. In the analysis of the longitudinal dimension, the spleen length of $>20 \mathrm{~cm}$ showed a statistically significant test for recurrence of variceal and a length $>19 \mathrm{~cm}$ presented as a very sensitive and statistically significant test for rebleeding CONCLUSION: Splenic index and craniocaudal dimension analysis, obtained by ultrasonography, can predict recurrence of varicose veins and rebleeding after exclusive endoscopic treatment.

HEADINGS: Schistosomiasis. Hypertension, Portal. Esophageal and Gastric Varices. Hypersplenism. Endoscopy, Gastrointestinal

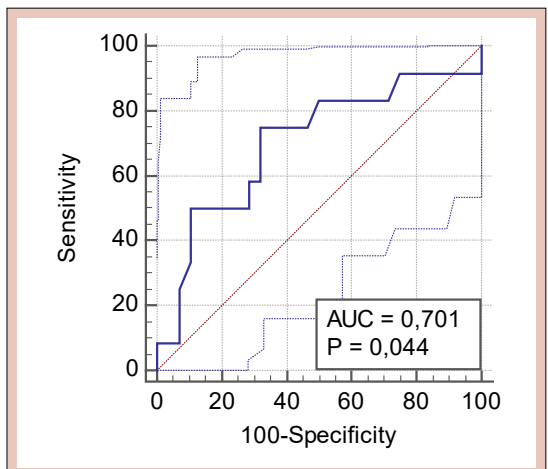

Figura 2 - Curva ROC - Índice esplênico médio para sangramento em pacientes esquistossomóticos com varizes esofágicas erradicadas por tratamento endosópico exclusivo

\begin{tabular}{l} 
Mensagem central \\
\hline O índice esplênico e a análise craniocaudal \\
unidimensional do baço, obtidos por \\
ultrassonografia, podem predizer recorrência \\
de varizes esofágicas e ressangramento após \\
erradicação por tratamento endoscópico \\
exclusive.
\end{tabular}

\section{Perspectivas}

A análise do índice esplênico mostrou fortes evidências no planejamento terapêutico de pacientes esquistossomóticos com hipertensão portal.

Como citar esse artigo: Borgheresi A, Colleoni R, Milton Scalabrini, Shigueoka D. O índice esplênico como preditor de sangramento e recidiva varicosa no seguimento tardio de paciente esquistossomóticos após tratamento endoscópico exclusivo. ABCD Arq Bras Cir Dig. 2021;34(4):e1638. https://doi.org/10.1590/0102$672020210002 \mathrm{e} 1638$

Correspondência:

Alexandre Borgheresi

alexandreborgheresi@hotmail.com
Fonte de financiamento: Não.

Conflito de interesses: Não.

Recebido:04/08/2021

Aceito: 30/09/2021 


\section{INTRODUÇÃO}

A esquistossomose é uma doença parasitária, que apresenta manifestações agudas e crônicas, causada por vermes trematódeos do gênero Schistosoma. Estimativas mostram que anualmente 220,8 milhões de pessoas no mundo precisam de tratamento preventivo ${ }^{10,14,24}$. No Brasil, a parasitose é causada exclusivamente pelo Schistosoma mansoni e representa um importante impacto na saúde pública, estima-se que tenhamos 4 milhões de pessoas infectadas e 20 milhões expostas à infecção ${ }^{14}$. A forma mais grave da doença, a hepatoesplênica, é uma importante causa de morbimortalidade em 3 a 10\% dos infectados ${ }^{8,11,21}$. O envolvimento hepatoesplênico crônico descreve uma ampla gama de manifestações clínicas importantes, sendo a hipertensão portal o principal fator de morbimortalidade nesses pacientes. Com a evolução crônica da doença, esses pacientes podem desenvolver ascite, esplenomegalia, trombose portal, varizes de esôfago e estômago, sendo a hemorragia digestiva alta a principal causa de mortalidade ${ }^{2,3,4,15,18}$. Por esse motivo, o desenvolvimento de métodos para o diagnóstico precoce das complicações e o tratamento eficaz são prioridades na literatura médica atual.

O tratamento endoscópico das varizes esofágicas tem sido utilizado como principal intervenção em pacientes com hipertensão portal secundária à esquistossomose. A avaliação dos resultados em longo prazo do tratamento endoscópico exclusivo nesse grupo de pacientes ainda é insuficiente e controversa. Estudos já demonstraram a importância dos achados da ultrassonografia com doppler no diagnóstico da esquistossomose, além da reprodutibilidade adequada desse método, mas ainda faltam informações sobre o valor prognóstico e aplicabilidade clínica desses achados. Assim, é importante estudar o comportamento desta doença e os valores do índice esplênico como preditor de resposta ao tratamento para melhorar a qualidade da assistência, reduzindo a mortalidade e morbidade.

O objetivo deste estudo foi avaliar o seguimento a longo prazo de pacientes com esquistossomose hepatoesplênica submetidos ao tratamento endoscópico exclusivo com erradicação das varizes esofágicas por análise das dimensões ultrassonográficas do baço e sua relação com a recorrência de varizes esofágicas e ressangramento.

\section{MÉTODOS}

\section{Amostra e desenho do estudo}

Trata-se de um estudo observacional, retrospectivo, por meio da análise de prontuários de pacientes com diagnóstico de esquistossomose hepatoesplênica, em acompanhamento no Ambulatório do Grupo de Gastroenterologia Cirúrgica da Escola Paulista de Medicina - UNIFESP, no período de $1^{\circ}$ de janeiro de 2002 a $1^{\circ}$ de janeiro de 2019 .

Os pacientes incluídos foram submetidos ao tratamento endoscópico exclusivo com erradicação das varizes esofágicas por ligadura elástica e / ou escleroterapia, o mesmos foram acompanhados anualmente após a erradicação das varizes com ultrassonografia, endoscopia digestiva alta, exames laboratoriais e história clínica. Este estudo foi realizado de acordo com a Declaração de Helsink e aprovado pelo Comitê de Ética em Pesquisa da UNIFESP $\left(n^{\circ}=2462087\right)$.

Os critérios de elegibilidade incluíram pacientes com seguimento mínimo de 12 meses após a erradicação das varizes esofágicas detectadas por exame endoscópico e disponibilidade de exames laboratoriais, endoscopicos e ultrassonografia abdominal com doppler. Foram excluídos pacientes com hepatopatia de outra etiologia, pacientes submetidos à cirurgia (esplenectomia) antes da erradicação endoscópica e ausência do laudo da dimensão longitudinal ou doíndice esplênico nos exames ultrassonográficos.

\section{Variáveis}

Os dados epidemiológicos foram coletados de forma padronizada e tabulados. Todos os pacientes foram avaliados retrospectivamente por ultrassonografia doppler, endoscopia digestiva alta, exames laboratoriais e história clínica, realizados em série durante o período de acompanhamento.

As informações ultrassonográficas foram avaliadas e tabuladas de acordo com o protocolo de Niamey, adotado como padronização pela OMS na avaliação de exames de esquistossomóticos ${ }^{1,3}$. Foram avaliados os seguintes parâmetros: calibre e fluxo da veia porta e veia esplênica, transformação cavernomatosa, índice esplênico, presença de nódulos sideróticos (corpúsculo Gamma Gandhi), tamanho do baço e ascite. Em relação aos exames laboratoriais, foram avaliados os seguintes fatores: leucograma, hemoglobina, bilirrubinas, INR, APTT, albumina, contagem de plaquetas e enzimas hepáticas. Valores de hemoglobina menores que $12 \mathrm{~g} / \mathrm{I}$, leucócitos menores que 3500 , TGO maior que 32, TGP maior que 33, albumina menor que $3,5, \mathrm{RNI}$ maior que 1,2 , plaquetas menores que $150.000 \mathrm{e}$ bilirrubinas totais maiores que 1 foram considerados alterados ${ }^{19}$.

Os dados endoscópicos analisados foram: recorrência de varizes esofágicas, calibre e número de varizes na recorrência, necessidade de novas sessões de tratamento após a erradicação, tipo de tratamento realizado e ressangramento. Os parâmetros de evolução clínica avaliados: mortalidade, necessidade de cirurgia para hipertensão portal durante o seguimento e sangramento após erradicação.

\section{Análise estatística}

Para a análise descritiva, foram utilizadas variáveis categóricas de frequência e porcentagem. Para as variáveis contínuas, foram utilizados média, desvio padrão, mínimo, mediana e máximo. Para a análise de correlação e curva ROC, foi utilizado o valor médio do índice esplênico e da dimensão longitudinal de cada paciente. Os pacientes que não fizeram uma avaliação ultrassonográfica do valor do índice esplênico ou da dimensão longitudinal do baço foram excluídos dessas análises. Para determinar o melhor ponto de corte para o índice esplênico médio que previa recorrência e sangramento, foi usada a curva ROC. A precisão foi determinada a partir da área sob a curva ROC usando o programa SPSS 17.0. Foram analisados a sensibilidade, especificidade, valor preditivo positivo (VP +) e valor preditivo negativo (VP -) das variáveis indicativas de recorrência e sangramento. Para comparar o índice esplênico com variáveis categóricas, foi utilizado o teste t. A análise estatística foi realizada por meio do programa SAS 9.1, com nível de significância de $5 \%$.

\section{RESULTADOS}

Foram analisados prontuários de 44 pacientes (feminino = 22; masculino $=22$ ), os quais continham a descrição do índice esplênico e / ou dimensão longitudinal do baço. A média de idade dos pacientes no tempo zero foi de 49,75 anos, com um mínimo de 31 e um máximo de 79 anos. O tempo médio de acompanhamento foi de 8,07 anos, com mínimo de 1 e máximo de 16 anos (Tabela 1). Em relação aos exames, foram analisados um total de 150 ultrassonografias doppler de abdome, 245 endoscopias digestivas altas e 76 exames laboratoriais.

Em relação aos desfechos primários, $63,6 \%$ apresentaram recorrência de varizes esofágicas após a erradicação. $O$ tempo médio para a primeira recidiva foi de 2,32 anos, com uma média de 2,07 recidivas no período de acompanhamento. (Tabela 2) $29,5 \%$ dos pacientes apresentaram sangramento gastrointestinal após a erradicação, em 76,9\% dos casos, sangramento com repercussões hemodinâmicas ou hematimétricas. 
A terapia mais comumente utilizada para o controle da erradicação após a recorrência e sangramento foi a ligadura elástica. (Tabela 3).

A mortalidade por hemorrragia digestiva alta (HAD) foi de $6,8 \%$ durante o seguimento. As demais causas são apresentadas na tabela a seguir (Tabela 4).

Tabela 1 - Distribuição de acordo com a idade e o tempo de seguimento dos pacientes esquistossomóticos com varizes de esôfago erradicadas por tratamento endosópico exclusivo.

$\begin{array}{lc}\text { Idade na erradicação (anos) } & \text { Total } \\ \text { Média (SD) } & 49.75(11.61) \\ \text { Mediana } & 48 \\ \text { Mínimo e Máximo } & 31-79 \\ \text { Total pacientes } & 44 \\ \text { Tempo seguimento (anos) } & \\ \text { Média (SD) } & 8.07(12.51) \\ \text { Mediana } & 6 \\ \text { Mínimo e Máximo } & 1-16 \\ \text { Total pacientes } & 44\end{array}$

Tabela 2 - Descrição dos achados endoscópicos em relação à recidiva de varizes em pacientes com esquistossomose com varizes de esôfago erradicadas por tratamento endosópico exclusivo.

$\begin{array}{lc}\text { Recidiva } & \text { Total } \\ \text { Não } & 16(36.4 \%) \\ \text { Sim } & 28(63.6 \%) \\ \text { Total pacientes } & 44 \\ \text { Número de recidiva } & 2.07(1.54) \\ \text { Média (SD) } & 1 \\ \text { Mediana } & 1-6 \\ \text { Mínimo Máximo } & 28 \\ \text { Total pacientes } & \\ \text { Número médio de recidiva varicosa } & 2.42(1.01) \\ \text { Média (SD) } & 2.325 \\ \text { Mediana } & 1-6 \\ \text { Mínimo e Máximo } & 28 \\ \text { Total pacientes } & 2.32(2.36) \\ \text { Tempo médio para primeira recidiva } & 1 \\ \text { Média (SD) } & 1-9 \\ \text { Mediana } & \\ \text { Mínimo e Máximo } & 28 \\ \text { Total pacientes } & \end{array}$

Tabela 3 - Descrição dos achados endoscópicos em relação ao ressangramento em pacientes esquistossomóticos com varizes de esôfago erradicadas por tratamento endosópico exclusivo

$\begin{array}{lc} & \text { Total } \\ \text { Sangramento } & \\ \text { Não } & 31(70.5 \%) \\ \text { Sim } & 13(29.5 \%) \\ \text { Total pacientes } & 44 \\ \text { Tipo de tratamento } & \\ \text { Ligadura elástica } & 21(8.6 \%) \\ \text { Escleroterapia } & 13(5.3 \%) \\ \text { Total exames } & 245 \\ \text { Repercussão hematimétrica ou hemodinamica } \\ \text { Não } \\ \text { Sim } & 3(23 \%) \\ & 10(76.9 \%)\end{array}$

A análise descritiva dos achados ultrassonográficos mostrou que o calibre médio da veia porta era de $1,39 \mathrm{~cm}$ e da veia esplênica de $1,26 \mathrm{~cm}$. A presença de ascite foi identificada em $14,7 \%$ dos exames, sendo $59 \%$ pequena (Tabela 5 ).

Fluxo hepatofugal foi observado em $9 \%$ dos pacientes. O tamanho do baço (longitudinal) foi descrito em 23 pacientes, sendo o valor médio de $20,16 \mathrm{~cm}$. O índice esplênico foi descrito em 40 pacientes, com média de 151,6. A transformação cavernomatosa foi identificada em $38,6 \%$ dos pacientes. $47,7 \%$ dos pacientes apresentavam nódulos sideróticos nos achados ultrassonográficos. (Tabela 6)

Os achados laboratoriais mostraram que o valor médio do leucograma foi de 4.152, com 57,1\% dos pacientes leucopênicos. O valor médio das plaquetas nos pacientes foi $73.333,88 \%$ plaquetopênicos. $28,6 \%$ dos pacientes apresentavam anemia. (Tabela 7)

A análise da curva ROC do índice esplênico médio por recorrência de varizes demonstrou um valor de corte $>169$,

Tabela 4 - Descrição da mortalidade durante o seguimento de pacientes com esquistossomose e varizes de esôfago erradicadas por tratamento endoscópico exclusivo

$\begin{array}{lc}\text { Mortalidade } & \text { Total } \\ \text { Não } & 34(77.3 \%) \\ \text { Sim } & 10(22.7 \%) \\ \text { Total pacientes } & 44 \\ \text { Mortalidade por causa } & \\ \text { Sangramento pós operatório } & 1 \\ \text { Desconhecidas } & 3 \\ \text { HDA } & 3(30 \%) \\ \text { Insuficiência Cardiaca } & 1 \\ \text { Pneumonia Nosocomial } & 1 \\ \text { Peritonite bacteriana espontânea } & 1 \\ \text { Total pacientes } & 10 \\ \text { Mortalidade por HDA } & \\ \text { Não } & 41(93.2 \%) \\ \text { Sim } & 3(6.8 \%) \\ \text { Total pacientes } & 44\end{array}$

Tabela 5 - Descrição dos achados ultrassonográficos em pacientes com esquistossomose com varizes de esôfago erradicadas por tratamento endosópico exclusivo

$\begin{array}{lc}\text { Calibre Portal } & \text { Total } \\ \text { Média (SD) } & 1.39(0.38) \\ \text { Mediana } & 1.4 \\ \text { Minimo Maximo } & 0.4-2.4 \\ \text { Total pacientes } & 44 \\ \text { Calibre Veia Esplênica } & \\ \text { Média (SD) } & 1.26(0.38) \\ \text { Mediana } & 1.2 \\ \text { Minimo Maximo } & 0.6-2.4 \\ \text { Total pacientes } & 44 \\ \text { Ascite } & 128(85.3 \%) \\ \text { Não } & 22(14.7 \%) \\ \text { Sim } & 150 \\ \text { Total exames } & \\ \text { Ascite - Volume } & 2(9 \%) \\ \text { Grande } & 7(31.8 \%) \\ \text { Moderada } & 13(59 \%) \\ \text { Pequena } & 22 \\ \text { Total exames } & \\ & \end{array}$


Tabela 6 - Descrição dos achados ultrassonográficos em pacientes esquistossomóticos com varizes de esôfago erradicadas por tratamento endoscópico exclusivo

\begin{tabular}{lc}
\hline Hepatofugal & Total \\
Não & $39(88.6 \%)$ \\
Sim & $4(9 \%)$ \\
Total exames & 44 \\
Média dimensão longitudinal do baço & \\
Média (SD) & $20.16(2.63)$ \\
Mediana & 20 \\
Minimo Maximo & $15-24.2$ \\
Total pacientes & 23 \\
Média índice esplênico & \\
Média (SD) & $151.62(41.91)$ \\
Mediana & 142.25 \\
Minimo Maximo & $76-221.5$ \\
Total pacientes & 40 \\
Transformação cavernomatosa & \\
Não & $27(61.3 \%)$ \\
Sim & $17(38.6 \%)$ \\
Total exames & 44 \\
Nódulo siderótico & \\
Não & $23(52.2 \%)$ \\
Sim & $21(47.7 \%)$ \\
Total exames & 44 \\
\end{tabular}

determinando um VP + de 73\% de recorrência para valores maiores que 169 (AUC =0,57, $p=0,42$ ) (Figura 1, Tabela 8)

A curva ROC do índice esplênico médio por sangramento mostrou que o valor $>144$ foi observado como ponto de corte, determinando uma sensibilidade de $75 \%$ com VP- de $86 \%$ de sangramento para valores maiores que 144 ( $A \cup C=0,70$, $p=0,044$ ) (Figura 2, Tabela 9).

A curva ROC do tamanho médio do baço para recorrência de varizes demonstrou um valor de corte $>20$, determinando uma especificidade de $100 \%$ com VP + de $100 \%$ de recorrência para valores maiores que 20 (AUC 0,71, $p=0,047$ ) (Figura 3 , Tabela 10).

A curva ROC da média do tamanho do baço para sangramento teve um valor de corte de $>19$, determinando uma sensibilidade de $100 \%$ com VP- de $100 \%$ de sangramento para valores maiores que 19 (AUC 0,76, $p=0,008$ ) (Figura 4, Tabela 11)

Por fim, na análise do cruzamento das variáveis categóricas com o índice esplênico, houve significancia estatistica na analise do índice esplênico e necessidade de cirurgia. Os pacientes operados apresentaram índice médio mais elevado. $(P=0,0021)$ Não houve diferença estatisticamente significativa nas demais variáveis estudadas (Tabela 12, Figura 5).

\section{DISCUSSÃO}

Os principais achados deste estudo, apontaram que o índice esplênico e a análise craniocaudal unidimensional do baço, obtidos pela ultrassonografia, podem predizer a ocorrência de recicidiva de varizes esofágicas e ressangramento após erradicação endoscópica exclusiva, contribuindo para o planejamento terapêutico dos esquistossomóticos com hipertensão portal.

A esquistossomose é endêmica em uma vasta área do país, ainda considerada um grave e negligenciado problema de saúde pública, pois atinge milhões de pessoas, causando um número expressivo de formas graves e óbitos ${ }^{13}$. A redução da
Tabela 7 - Descrição dos achados laboratoriais em pacientes esquistossomóticos com varizes de esôfago erradicadas por tratamento endoscópico exclusivo

\begin{tabular}{|c|c|}
\hline & Total \\
\hline \multicolumn{2}{|l|}{ Leucograma } \\
\hline Média (SD) & 4152.38 (2395.98) \\
\hline Mediana & 3235 \\
\hline Minimo Maximo & $1490-11600$ \\
\hline Total pacientes & 44 \\
\hline \multicolumn{2}{|l|}{ Interpretação } \\
\hline Normal & 18 (42.9\%) \\
\hline Alterado & $24(57.1 \%)$ \\
\hline \multicolumn{2}{|l|}{ Plaquetas } \\
\hline Média (SD) & $73333.33(58981.46)$ \\
\hline Mediana & 53000 \\
\hline Minimo Maximo & $18000-241000$ \\
\hline Total pacientes & 44 \\
\hline \multicolumn{2}{|l|}{ Interpretação } \\
\hline Normal & 5 (11.9\%) \\
\hline Alterado & 37 (88.1\%) \\
\hline \multicolumn{2}{|l|}{ Bilirrubinas } \\
\hline Média (SD) & $1.6(1.26)$ \\
\hline Mediana & 1.16 \\
\hline Minimo Maximo & $0.4-4.8$ \\
\hline Total pacientes & 35 \\
\hline \multicolumn{2}{|l|}{ Interpretação } \\
\hline Normal & $14(40 \%)$ \\
\hline Alterado & $21(60 \%)$ \\
\hline \multicolumn{2}{|l|}{ RNI } \\
\hline Média (SD) & $1.37(0.28)$ \\
\hline Mediana & 1.3 \\
\hline Minimo Maximo & $1-2.22$ \\
\hline Total pacientes & 39 \\
\hline \multicolumn{2}{|l|}{ Interpretação } \\
\hline Normal & $10(25.6 \%)$ \\
\hline Alterado & 29 (74.4\%) \\
\hline \multicolumn{2}{|l|}{ Hemoglobina } \\
\hline Média (SD) & $12.04(2.09)$ \\
\hline Mediana & 12 \\
\hline Minimo Maximo & 06 \\
\hline Total pacientes & 44 \\
\hline \multicolumn{2}{|l|}{ Interpretação } \\
\hline Normal & 30 (71.4\%) \\
\hline Alterado & $12(28.6 \%)$ \\
\hline \multicolumn{2}{|l|}{ TGO } \\
\hline Média (SD) & $39.63(23.46)$ \\
\hline Mediana & 33 \\
\hline Minimo Maximo & $16-150$ \\
\hline Total pacientes & 38 \\
\hline \multicolumn{2}{|l|}{ Interpretação } \\
\hline Normal & $19(50 \%)$ \\
\hline Alterado & $19(50 \%)$ \\
\hline \multicolumn{2}{|l|}{ TGP } \\
\hline Média (SD) & $32.78(10.65)$ \\
\hline Mediana & 32 \\
\hline Minimo Maximo & 60 \\
\hline Total pacientes & 37 \\
\hline \multicolumn{2}{|l|}{ Interpretação } \\
\hline Normal & 22 (59.5\%) \\
\hline Alterado & $15(40.5 \%)$ \\
\hline \multicolumn{2}{|l|}{ Albumina } \\
\hline Média (SD) & $3.55(0.87)$ \\
\hline Mediana & 3.7 \\
\hline Minimo Maximo & $0.7-4.9$ \\
\hline Total pacientes & 35 \\
\hline \multicolumn{2}{|l|}{ Interpretação } \\
\hline Normal & 22 (62.9\%) \\
\hline Alterado & $13(37.1 \%)$ \\
\hline
\end{tabular}




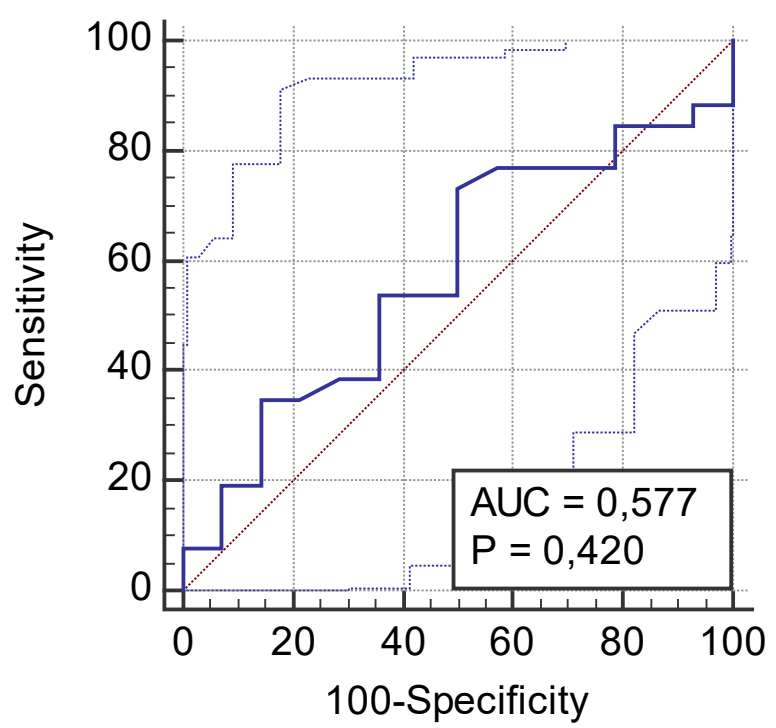

Figura 1 - Curva ROC - Índice esplênico médio e recorrência de varizes em pacientes esquistossomóticos com varizes de esôfago erradicadas por tratamento endosópico exclusivo

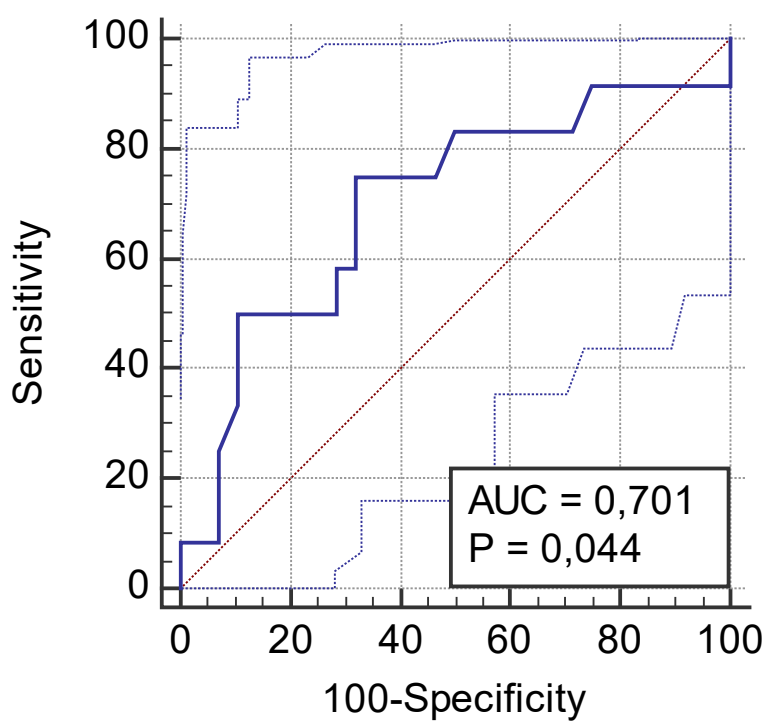

Figura 2 - Curva ROC - Índice esplênico médio para sangramento em pacientes esquistossomóticos com varizes esofágicas erradicadas por tratamento endosópico exclusivo

Tabela 8 - Teste diagnóstico do índice esplênico médio para recidiva de varizes em pacientes com esquistossomose com varizes de esôfago erradicadas por tratamento endosópico exclusivo

\begin{tabular}{|c|c|c|c|c|c|}
\hline & \multicolumn{2}{|c|}{ Sangramento } & \multirow{2}{*}{ Total } & \multirow{2}{*}{$\begin{array}{l}\text { VP+ } \\
\text { VP- }\end{array}$} & \multirow{2}{*}{$\begin{array}{l}0.50 \\
0.86\end{array}$} \\
\hline & Sim & Não & & & \\
\hline Índice esplênico & & & & sensibilidade & 0.75 \\
\hline $\operatorname{Sim}(>140)$ & 9 (22.5\%) & 9 (22.5\%) & $18(45 \%)$ & especificidade & 0.68 \\
\hline Não $(\leq 140)$ & $3(7.5 \%)$ & $19(47.5 \%)$ & 22 (55\%) & acurácia & 0.70 \\
\hline Total & $12(30 \%)$ & 28 (70\%) & 40 (100\%) & prevalência & 0.30 \\
\hline
\end{tabular}

Tabela 9 - Teste diagnóstico do índice esplênico médio para sangramento em pacientes esquistossomóticos com varizes de esôfago erradicadas por tratamento endoscópico exclusivo

\begin{tabular}{|c|c|c|c|c|c|}
\hline & \multicolumn{2}{|c|}{ Recidiva } & \multirow{2}{*}{ Total } & \multirow{2}{*}{$\begin{array}{l}\text { VP+ } \\
\text { VP- }\end{array}$} & \multirow{2}{*}{$\begin{array}{l}0.73 \\
0.50\end{array}$} \\
\hline & Sim & Não & & & \\
\hline Índice esplênico & & & & sensibilidade & 0.73 \\
\hline $\operatorname{Sim}(>169)$ & 19 (47.5\%) & 7 (17.5\%) & $26(65 \%)$ & especificidade & 0.50 \\
\hline Não ( $\leq 169)$ & 7 (17.5\%) & 7 (17.5\%) & $14(35 \%)$ & acurácia & 0.65 \\
\hline Total & $26(65 \%)$ & 14 (35\%) & 40 (100\%) & prevalência & 0.65 \\
\hline
\end{tabular}

morbidade e mortalidade da esquistossomose requer a detecção precoce e o tratamento imediato de todos os portadores para evitar que a ação patogênica acumulada dos ovos de S. mansoni cause alterações nos órgãos afetados. Porém, são poucos os locais que possuem centro de referência no tratamento e acompanhamento desses pacientes.

O estudo foi realizado em um Hospital Universitário que possui Ambulatório com especialistas que realizam o acompanhamento longitudinal dessa doença.

O número limitado de pacientes utilizado na amostra deve-se aos rígidos critérios de inclusão. Foram avaliados apenas os pacientes com varizes erradicadas que não haviam sido submetidos à esplenectomia no início do seguimento proposto. No entanto, foram avaliados retrospectivamente por um longo período de acompanhamento, com duração média de 8 anos, possibilitando a coleta de informações periódicas de um número significativo de laboratórios, exames endoscópicos e ultrassonográficos.

Conforme já demonstrado na literatura, não há diferença entre os gêneros, como também apontado por este estudo com igual proporção entre os gêneros ${ }^{13,16}$. A forma hepatoesplênica compensada predomina em adolescentes e adultos jovens, entre 10 e 30 anos. Nosso estudo mostrou uma média de idade superior, isso devido ao fato de ter sido selecionado um grupo de pacientes que estavam em acompanhamento clínico há algum tempo, com varizes erradicadas e compensadas no tempo de análise inicial. A idade ao diagnóstico não foi levada em consideração.

O sangramento gastrointestinal alto secundário à ruptura de varizes é uma das principais complicações da hipertensão portal devido à esquistossomose, ocorrendo em cerca de $30 \%$ a $40 \%$ dos pacientes ${ }^{13,20}$. Apesar dos avanços 
na terapia endoscópica, a taxa de mortalidade para um único episódio de sangramento por varizes é de $20 \%{ }^{5,13}$. Em nosso estudo, 6,8\% dos pacientes acompanhados morreram de hemorragia digestiva após erradicação das varizes. Para a prevenção secundária da hemorragia varicosa em pacientes com hipertensão portal esquistossomótica, temos a endoscopia como a técnica mais realizada, com eficácia relatada da escleroterapia ou ligadura elástica endoscópica variando de $54 \%$ a $82,3 \%{ }^{7}$. Em nossa amostra, a ligadura elástica foi a técnica mais utilizada tanto para a erradicação quanto para o controle do sangramento.

Mesmo com os avanços da endoscopia, de acordo com os escassos dados da literatura sobre o assunto, encontramos taxa de até $62 \%$ de recorrência de varizes de esôfago após tratamento endoscópico e taxa de ressangramento de $46 \%$ 9,22 . Em nossa série, os dados são semelhantes em relação à recorrência $(63,6 \%)$ e menores em relação a ressangramento (29,5\%). No entanto, é importante destacar que a maioria dos pacientes com ressangramento teve repercussões hematimétricas ou hemodinâmicas. Não encontramos na literatura, para uma análise comparativa, estudos que avaliem especificamente as características da recidiva, conforme demonstrado em nossa pesquisa. No entanto, consideramos 2,32 anos de intervalo livre de varizes médio como um tempo curto e consideramos expressivos 2,07 episódios de recorrência em média por período de acompanhamento.

De acordo com as normas técnicas vigentes do Ministério da Saúde, o tratamento cirúrgico pode ser considerado nas

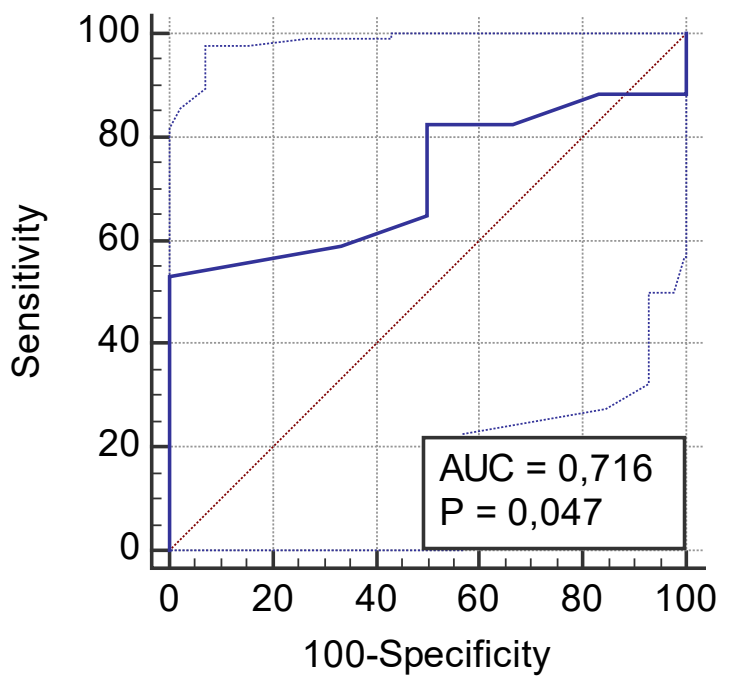

Figura 3 - Curva ROC - Tamanho médio do baço para recidiva de varizes em pacientes esquistossomóticos com varizes de esôfago erradicadas por tratamento endosópico exclusivo seguintes situações: 1 . varizes com sinais de sangramento iminente à endoscopia; 2 . grandes varizes gástricas e esofágicas em pacientes que vivem fora de grandes centros médicos; 3. Varizes gástricas grandes persistentes após erradicação endoscópica das varizes esofágicas; 4 . hiperesplenismo com manifestação clínica incapacitante ${ }^{14}$. Nas demais condições, os pacientes deveriam ser avaliados periodicamente e, nos casos de evolução desfavorável, seria indicado tratamento cirúrgico complementar. Durante o seguimento avaliado em nosso estudo, $13 \%$ dos pacientes foram submetidos à esplenectomia seguindo os critérios descritos acima. Esses pacientes que se submeteram à cirurgia, apresentaram maiores valores de índice esplênico ( $P=0,0021$ ), demonstrando correlação positiva entre as dimensões do baço e a necessidade de indicação do procedimento (Tabela 11, Figura 5). $O$ achado de um aumento significativo do volume esplênico pode ser considerado um critério para justificar o tratamento cirúrgico nesses pacientes por meio de um procedimento de desconexão azigoportal ou shunt esplenorrenal distal. Não encontramos na literatura estudos que analisem a taxa de indicação cirúrgica em pacientes com varizes erradicadas para uma análise comparativa. As alterações observadas nos resultados dos exames laboratoriais para avaliação da função hepática não foram exploradas, pois não estão relacionadas ao objetivo da pesquisa, porém, podemos analisar que uma parte considerável dos pacientes apresentou alterações nestes exames, inferindo-se evolução no seguimento tardio para a forma hepatoesplênica descompensada.

Buscamos identificar com este estudo um teste utilizando as dimensões do baço para identificar preditores de recorrência de varizes e sangramento, principal causa de morbimortalidade nesta doença. O índice esplênico mostrou-se um teste sensível a valores acima de 144 como preditor de sangramento (PV$85 \%, A U C=0,70, p=0,044$ ) e pode ser um teste útil quando apresenta valores abaixo de 144 (teste negativo). Em relação à recorrência de varizes, o índice esplênico maior que 169 , mostrou-se um teste inespecífico, com valor preditivo positivo de $73 \%$, sem significância estatística. Porém, na análise da dimensão craniocaudal, o baço acima de $20 \mathrm{~cm}$, mostrou-se um teste muito específico e estatisticamente significativo para recidiva (VP + de 100\%, AUC 0,71, $p=0,047)$. Por ser um teste específico, o valor acima de $20 \mathrm{~cm}$ (teste positivo) é um bom preditor de recorrência. Da mesma forma, o baço acima de $19 \mathrm{~cm}$, apresenta-se como um teste muito sensível e significativamente estatístico para sangramento (100\% VP-, AUC 0,76, p =0,008). O baço menor que $19 \mathrm{~cm}$ (teste negativo) apresenta-se como um teste preciso para descartar sangramento.

Em vista da falta de evidências sobre a terapia endoscópica exclusiva de longo prazo, algumas pesquisas mais atuais buscam avaliar os benefícios da esplenectomia associada. Série de casos indica que a combinação de terapia cirúrgica e endoscópica pode ser mais eficaz do que a terapia exclusiva ${ }^{6,17}$. Seguindo essa hipótese, um estudo retrospectivo mostrou que a escleroterapia endoscópica para controle da recidiva e erradicação foi mais eficaz em pacientes que já haviam sido submetidos a tratamento cirúrgico ${ }^{12,17,23}$. Costa Lacet e cols. ${ }^{4}$ demonstraram em estudo prospectivo e randomizado, maior

Tabela 10 - Teste diagnóstico de tamanho médio do baço para recidiva de varizes em pacientes com esquistossomose com varizes de esôfago erradicadas por tratamento endosópico exclusivo

\begin{tabular}{|c|c|c|c|c|c|}
\hline & \multicolumn{2}{|c|}{ Recidiva } & \multirow{2}{*}{ Total } & \multirow{2}{*}{$\begin{array}{l}\text { VP+ } \\
\text { VP- }\end{array}$} & \multirow{2}{*}{$\begin{array}{l}1.00 \\
0.43\end{array}$} \\
\hline & Sim & Não & & & \\
\hline Tamanho & & & & sensibilidade & 0.53 \\
\hline $\operatorname{Sim}(>20)$ & 9 (39.1\%) & $0(0 \%)$ & 9 (39.1\%) & especificidade & 1.00 \\
\hline Não ( $\leq 20)$ & 8 (34.8\%) & $6(26.1 \%)$ & $14(60.9 \%)$ & acurácia & 0.65 \\
\hline Total & 17 (73.9\%) & $6(26.1 \%)$ & 23 (100\%) & prevalência & 0.74 \\
\hline
\end{tabular}


sucesso na erradicação e controle da recidiva em pacientes submetidos à terapia combinada cirúrgica e endoscópica. No entanto, estudos foram realizados com o objetivo de avaliar a eficácia da erradicação e controle da recorrência de sangramento em estágio inicial do tratamento. Não temos

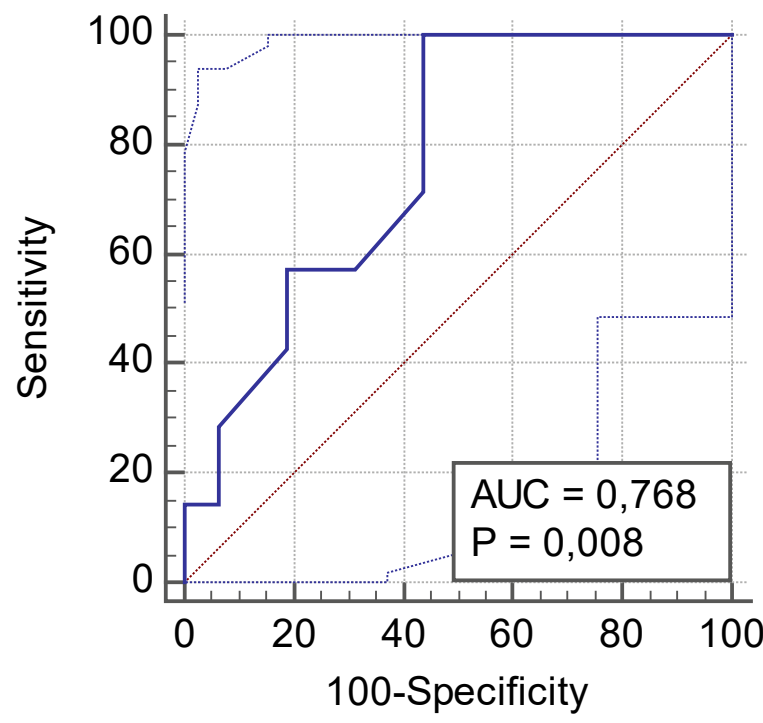

Figura 4 - Curva ROC - Tamanho médio do baço para sangramento em pacientes esquistossomóticos com varizes de esôfago erradicadas por tratamento endoscópico exclusivo estudos semelhantes avaliando desfechos tardios na população com varizes previamente erradicadas.

Em relação às limitações do estudo, podemos destacar o fato de ser retrospectivo e observacional em uma população limitada. Foi estudado um grupo específico de pacientes que tinham a característica de aderir ao tratamento e ao longo tempo de seguimento. Pacientes com esquistossomose geralmente apresentam características sociais que dificultam essa adesão, são originários de áreas endêmicas fora dos centros urbanos, geralmente possuem baixa renda e baixa escolaridade e, muitas vezes, mudam de endereço e de atividade profissional. Por isso,

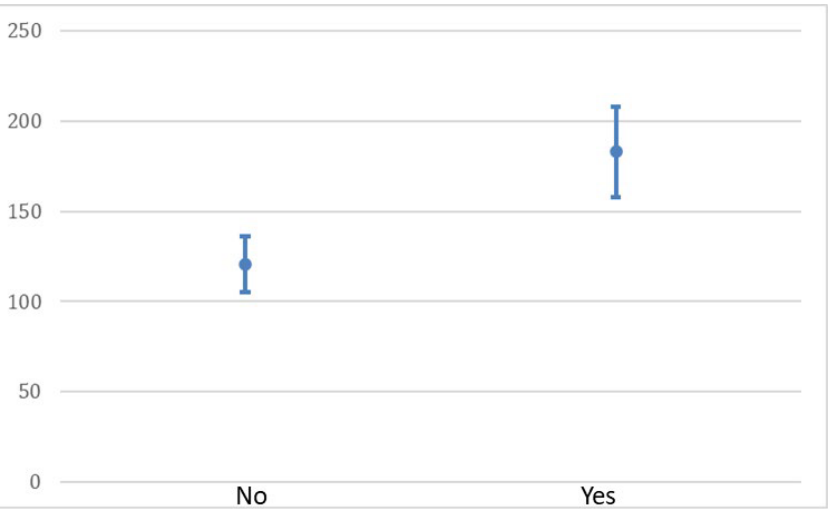

Figura 5 - Relação do índice esplênico com indicação de cirurgia em pacientes esquistossomóticos com varizes de esôfago erradicadas por tratamento endosópico exclusivo

Tabela 11 - Teste diagnóstico de tamanho médio do baço para sangramento em pacientes esquistossomóticos com varizes de esôfago erradicadas por tratamento endoscópico exclusivo

\begin{tabular}{|c|c|c|c|c|c|}
\hline & \multicolumn{2}{|c|}{ Sangramento } & \multirow{2}{*}{ Total } & \multirow{2}{*}{$\begin{array}{l}\text { VP+ } \\
\text { VP- }\end{array}$} & \multirow{2}{*}{$\begin{array}{l}0.50 \\
1.00\end{array}$} \\
\hline & Sim & Não & & & \\
\hline Tamanho & & & & sensibilidade & 1.00 \\
\hline $\operatorname{Sim}(>19)$ & 7 (30.4\%) & 7 (30.4\%) & 14 (60.9\%) & especificidade & 0.56 \\
\hline Não $(\leq 19)$ & $0(0 \%)$ & 9 (39.1\%) & 9 (39.1\%) & acurácia & 0.70 \\
\hline Total & 7 (30.4\%) & 16 (69.6\%) & $23(100 \%)$ & prevalência & 0.30 \\
\hline
\end{tabular}

Tabela 12 - Análise das variáveis categóricas em correlação com o índice esplênico médio de pacientes esquistossomóticos com varizes de esôfago erradicadas por tratamento endosópico exclusivo

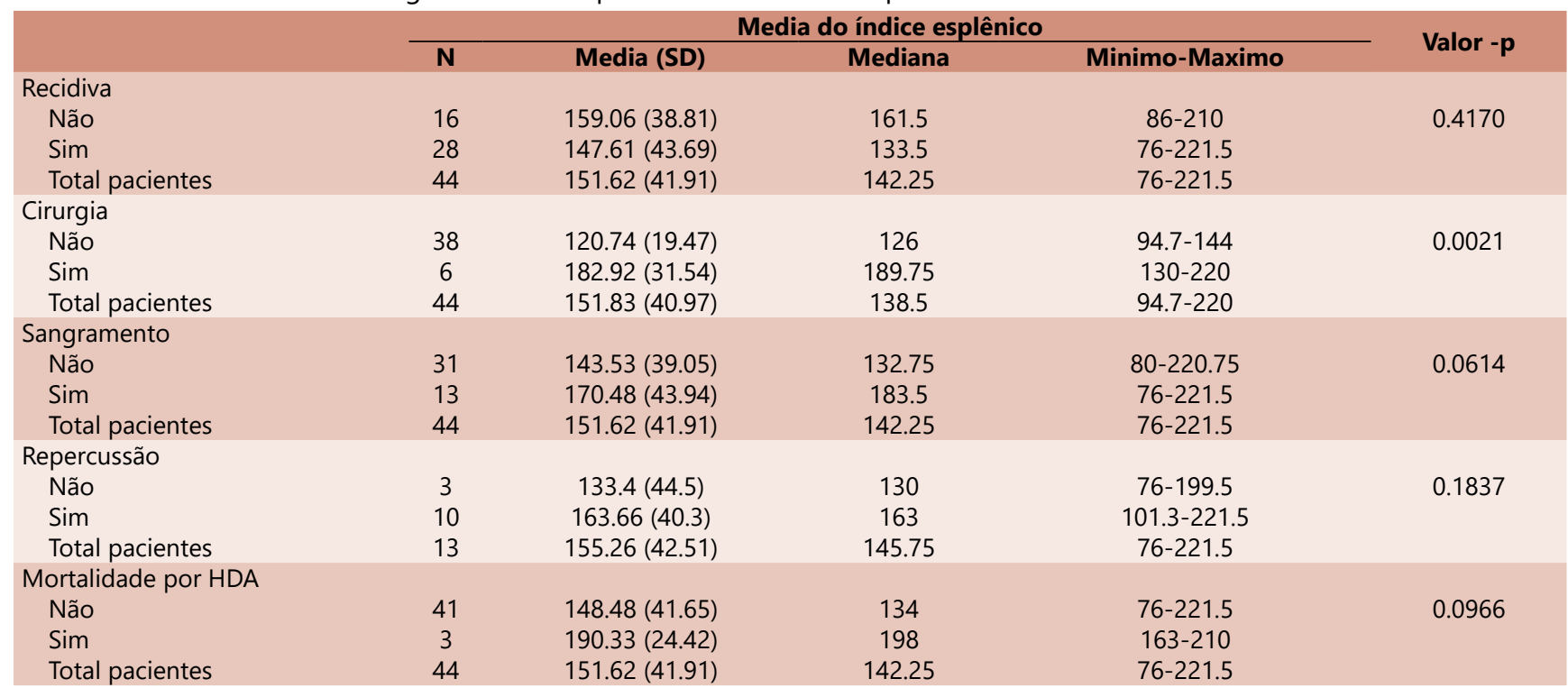


podemos ter apresentado viés de seleção. Podemos citar também como viés de aferição, a ausência do valor do índice esplênico ou dimensão craniocaudal em alguns exames avaliados. Para minimizar o viés de informação, todos os dados foram coletados de forma padronizada em tabelas por apenas um avaliador.

Por fim, a esquistossomose é uma doença ainda prevalente e relevante para a saúde pública em nosso país. A literatura atual é escassa em relação ao seguimento tardio desses pacientes, pouco se sabe sobre as consequências em longo prazo do tratamento endoscópico exclusivo. Os estudos ainda são controversos em relação à manutenção do baço, seu crescimento progressivo e as repercussões clínicas dele decorrentes. A análise periódica dos exames ultrassonográficos é recomendada na literatura e praticada em nosso país, mas sua aplicabilidade clínica é pouco conhecida. Estudos prospectivos e randomizados devem ser realizados para definir o melhor seguimento desses pacientes.

\section{CONCLUSÃO}

O índice esplênico e a análise craniocaudal unidimensional do baço, obtidos por ultrassonografia, podem predizer a ocorrência de recidiva de varizes esofágicas e ressangramento após erradicação endoscópica exclusiva, contribuindo para o planejamento terapêutico de pacientes esquistossomóticos com hipertensão portal.

\section{REFERÊNCIAS}

1. Abdel-Wahab MF, Esmat G, Milad M, Abdel-Razek S, Strickland GT. Characteristic sonographic pattern of schistosomal hepatic fibrosis. Am J Trop Med Hyg. 1989;40(1):72-6. doi: 10.4269/ ajtmh.1989.40.72

2. Bertoldi AS, Guetter CR, Coltro GA, Vosgerau LM, Brighenti LMV Fauat NI, Kubrusly FB, Marques CAM, Kubrusly LF. Carvedilol as primary prophylaxis for gastric variceal bleeding in portal hypertension model in rats. Arq Bras Cir Dig. 2020;33(3):e1525. doi: 10.1590/0102-672020200003e1525.

3. Batista-Neto J, Tognetti LB, Ribeiro LT, Balwani Mdo C, Muritiba $T$, Alves EE. Evolutional profile of the esophageal varices after splenectomy associated with ligation of the left gastric vein and sclerotherapy in schistosomal portal hypertension. Arq Bras Cir Dig. 2013;26(1):49-53. doi: 10.1590/s0102-67202013000100011.

4. Costa Lacet CM, Neto JB, Ribeiro LT, Oliveira FS, Wyszomirska RF, Strauss E. Schistosomal portal hypertension: Randomized trial comparingendoscopictherapyaloneorpreceded byesophagogastric devascularization and splenectomy. Ann Hepatol. 2016;15(5):73844. doi: 10.5604/16652681.1212528.

5. Coura JR, Conceição J, dos Santos ML, de MendonçaZG, Cutrim RN. Cross-sectional and evolutive studies of schistosomiasis mansoni in untreated and mass treated endemic areas in the southeast and northeast of Brazil. Mem Inst Oswaldo Cruz. 1992;87 Suppl 4:175-82. doi: 10.1590/s0074-02761992000800027.

6. Cordeiro F. Variceal sclerosis in schistosomotic patients: a 5-year follow-up study. Gastrointest Endosc. 1990;36(5):475-8. doi: 10.1016/s0016-5107(90)71118-2.

7. Domingues ALC. Schistosomiasis mansoni. In: Mattos AA,Dantas EB (Eds.). Tratado de Hepatologia. Vol. 1. Rio de Janeiro, Rubio, 2010, p. 301-16.

8. Ferreira FG, Chin EW, Santos Mde F, de Carvalho DL, De Capua Junior A. Portal congestion and thrombosis after esophagogastric devascularizationandsplenectomy].RevAssocMedBras.2005;51(4):2336. doi: 10.1590/s0104-42302005000400021.
9. Ferraz AA, Bacelar TS, Silveira MJ, Coelho AR, Câmara Neto RD, de Araújo Júnior JG, Ferraz EM. Surgical treatment of schistosomal portal hypertension. Int Surg. 2001;86(1):1-8. PMID: 11890333.

10. Global Health Estimates 2016: Deaths by Cause, Age, Sex, by Country and by Region, 2000-2016. Geneva, World Health Organization, 2018.

11. GBD2015Disease and Injury Incidenceand Prevalence Collaborators. Global, regional, and national incidence, prevalence, and years lived with disability for 310 diseases and injuries, 1990-2015: a systematic analysis for the Global Burden of Disease Study 2015. Lancet. 2016;388(10053):1545-1602. doi: 10.1016/S01406736(16)31678-6. Erratum in: Lancet. 2017;389(10064):e1.

12. Kiire CF. Controlled trial of propranolol to prevent recurrent variceal bleeding in patients with non-cirrhotic portal fibrosis. BMJ. 1989;298(6684):1363-5. doi: 10.1136/bmj.298.6684.1363.

13. Lambertucci JR. Revisiting the concept of hepatosplenic schistosomiasis and its challenges using traditional and new tools. Rev Soc Bras Med Trop. 2014;47(2):130-6. doi: 10.1590/00378682-0186-2013.

14. Ministério da Saúde, Vol.49- N49,2018-Situação Epidemiológica e estratégias de prevenção, controle e eliminação das Doenças Tropicais Negligenciadas no Brasil, 1995 a 2016.

15. MakdissiF.Trombosedosistemaportalnopós-operatóriodadesconexão ázigo-portal e esplenectomia em portadores e esquistossomose hepato-esplênica: avaliação de incidência, fatores preditivos, evolução clínica, laboratorial, endoscópica e de ultrassonografia com Doppler. Dissertação de Mestrado, FMUSP, 2004.

16. Martins RN, de Cleva R, Gouveia EM, Ghosn NB, Herman P. Correlation between splenomegaly and thrombocytopenia in hepatosplenic schistosomiasis. Arq Bras Cir Dig. 2010;23(4):254258. doi.org/10.1590/S0102-67202010000400010

17. PetroianuA,DeOliveiraAE, AlbertiLR. Hypersplenisminschistosomatic portal hypertension. Arch Med Res. 2005;36(5):496-501. doi: 10.1016/j.arcmed.2005.04.003.

18. Rebouças G. Clinical aspects of hepatosplenic schistosomiasis: a contrast with cirrhosis. Yale J Biol Med. 1975;48(5):369-76. PMID: 128911; PMCID: PMC2595267.

19. Richter, J, Hatz, Christopher, Campagne, G, Bergquist, N. R, Jenkins, Jennifer M. et al. (2000). Ultrasound in schistosomiasis: a practical guide to the standard use of ultrasonography for assessment of schistosomiasis-related morbidity. Second International Workshop, October 22-26 1996, Niamey, Niger / editors: J. Richter et al. Revised and updated. World Health Organization. https://apps. who.int/iris/handle/10665/66535

20. Rocha MO, Pedroso ER, Lambertucci JR, Greco DB, Rocha RL, Rezende DF, Neves J. Gastro-intestinal manifestations of the initial phase of schistosomiasis mansoni. Ann Trop Med Parasitol. 1995;89(3):271-8. doi: 10.1080/00034983.1995.11812952.

21. SecorWE, Colley DG. When Should the Emphasis on Schistosomiasis Control Move to Elimination. Trop Med Infect Dis. 2018;3(3):85. doi:10.3390/tropicalmed3030085.

22. Sakai P, Boaventura $S$, Ishioka $S$, Mies S, Sette H Jr, Pinotti HW. Sclerotherapy of bleeding esophageal varices in schistosomiasis. Comparative study in patients with and without previous surgery for portal hypertension. Endoscopy. 1990;22(1):5-7. doi:10.1055/s2007-1012777.

23. Sakai P. Esclerose endoscópica das varizes esofágicas após tratamento cirúrgico da hipertensão portal em pacientes com esquistossomose hepatoesplênica [Endoscopic sclerosis of esophageal varices after surgical treatment of portal hypertension in patient with hepatosplenic schistosomiasis]. Arq Gastroenterol. 2001;38(2):81-3. doi: 10.1590/s0004-28032001000200001.

24. Thomas AK, Dittrich M, Kardorff R, Talla I, Mbaye A, Sow S, Niang M, Yazdanpanah Y, Stelma FF, Gryseels B, Doehring E. Evaluation of ultrasonographic staging systems for the assessment of Schistosoma mansoni induced hepatic involvement. Acta Trop. 1997;68(3):347-56. doi: 10.1016/s0001-706x(97)00112-5. 